\title{
Passive acoustic monitoring of gunshot activity in CookingLake- Blackfoot provincial recreation area
}

Keelin Henderson Pekarik ${ }^{1}$, Justin Johnson ${ }^{1}$, Jeremiah Kennedy ${ }^{1}$, Richard Hedley ${ }^{1}$, Erin Bayne ${ }^{1}$

${ }^{1}$ Department Biological Sciences, University of Alberta

\begin{abstract}
In the past, monitoring hunting behavior has been limited to self-reported numbers. However, the ability of autonomous recording units to monitor soundscapes may make them suitable for assessing spatio-temporal shooting patterns. Our goal for this project was to find out if it is possible to use acoustic monitoring to track human activity, and if there were differences in seasonal or daily shooting intensities. We hypothesized that shooting intensity would decrease from September to November and from the afternoon till morning due to people being less likely to go shooting in cooler temperatures. A grid of 91 ARU's were deployed between September 2nd and November 30th, 2018 in Cooking Lake-Blackfoot Provincial Recreation Area. They were set to record continuously between sunrise and sunset with some recording during the night as well. We selected a random subset of 30 minute recordings, visualized them using spectrograms; visual representations of sound with time on the $\mathrm{x}$-axis and frequency on the $y$ axis, and counted the gunshots in each. We compared differences in gunshot detections between months and different times of day using analysis of variance (ANOVA). There were no statistical differences found in seasonal or daily shooting intensities. One reason for this may be that sample sizes were low, due to the time needed to manually process recordings. We demonstrated that ARU's can be used to provide us with an accurate way of assessing shooting patterns and therefore, be useful for monitoring other human behaviors such as detecting poachers, or assessing compliance with hunting laws.
\end{abstract}

Key words:

guns, gun shots, frequency, sound, sound recordings, hunting activity, acoustic monitoring, passive acoustic monitoring, ARU, ARU's, Autonomous recording unit, shooting, shooting intensity, shooting patterns, hunting patterns, Cooking Lake-Blackfoot Provincial Recreation Area, spectrograms, shot counts

Cite as: Henderson-Pekarik K., Johnson J., Kennedy J., Hedley R., Bayne E. 2019. Passive acoustic monitoring of gunshot activity in Cooking Lake-Blackfoot provincial recreation area. Alberta Academic Review, Vol 2 (2) 35-36, WISEST Special Issue (not peer-reviewed), DOI 10.29173/aar44. 
Henderson Pekarik et al., 2019

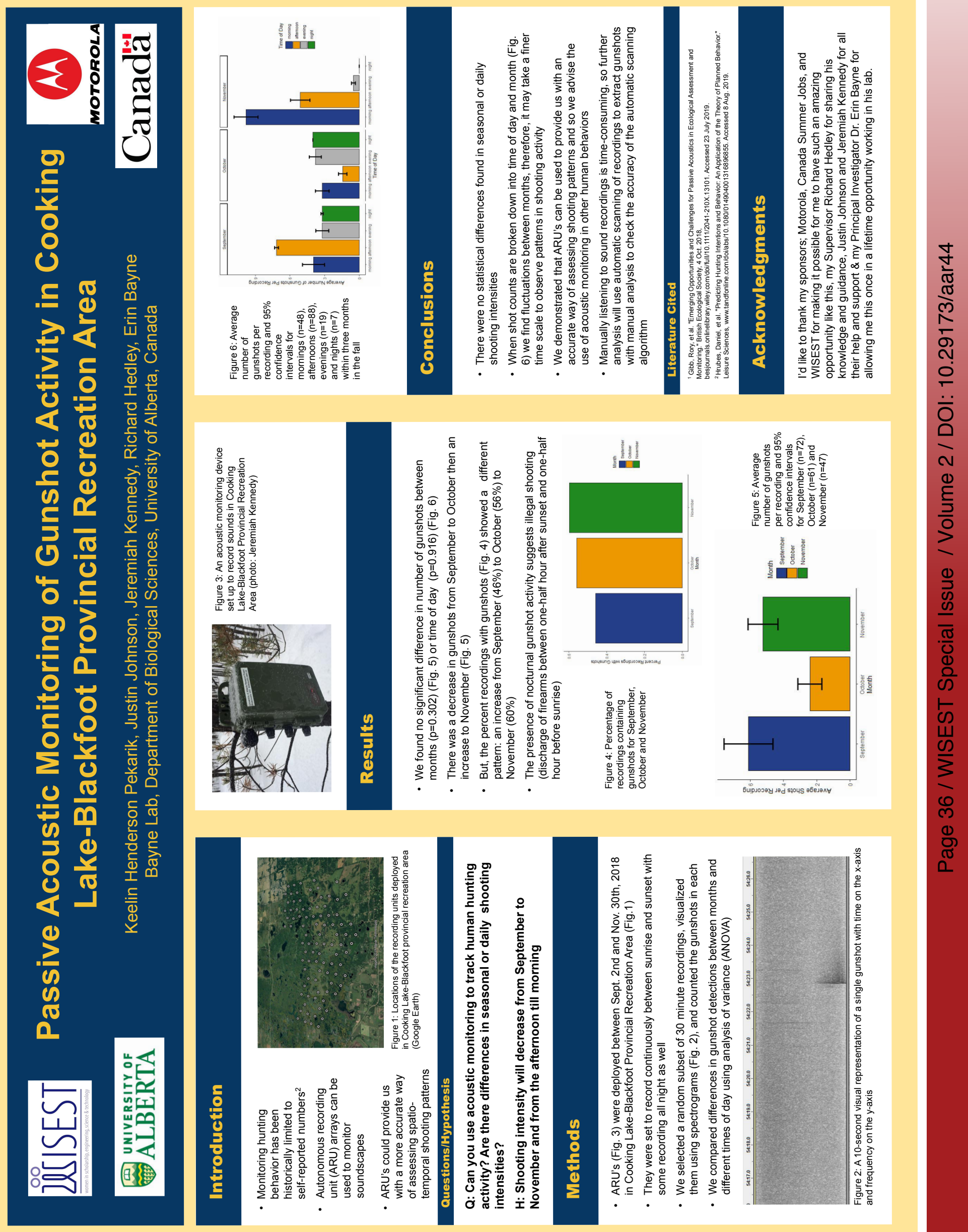

